\title{
Smartphone based ROP (S-ROP) screening-opportunities and challenges
}

\author{
Jayaprakash patil ${ }^{1}$ Laxmi patil ${ }^{1} \cdot$ Nikulaa Parachuri $^{2} \cdot$ Nilesh Kumar $^{1}{ }^{2} \cdot$ Francesco Bandello $^{3}$. \\ Baruch D. Kuppermann ${ }^{4}$. Anat Loewenstein ${ }^{5}$ - Ashish Sharma ${ }^{2}$
}

Received: 30 March 2020 / Revised: 16 April 2020 / Accepted: 17 April 2020 / Published online: 28 April 2020

(c) The Royal College of Ophthalmologists 2020

Devices such as the MII Ret Cam (MII Ret Cam Inc, Coimbatore, TN, India) have revolutionised smartphone based retinal imaging [1]. The affordability, portability and connectivity has popularised smartphone based retinal imaging beyond all boundaries. They are becoming increasingly popular in retinal imaging for patient records, academic purposes and also for medico-legal reasons. There are several studies using smart phones to image the retina in diabetic retinopathy (DR) screening and optic disc in glaucoma screening [1-3]. Ophthalmologists are now finding its utility in retinopathy of prematurity (ROP) screening. Review of literature reveals that there are several studies already in smartphone based retinopathy of prematurity (S-ROP) screening [4]. There are, however, certain limitations and challenges with S-ROP screening that has prevented it from being embraced by the ophthalmic fraternity as the mainstay tool in ROP screening. On a brighter note, the advanced imaging platforms with the newer smart phones and their adaptability has also opened up several opportunities for research and development. This has paved way for the integration with novel concepts of artificial intelligence (AI) and 3D printing technology [5-8].

$\triangle$ Ashish Sharma

drashish79@hotmail.com

1 University Hospital of Morecambe Bay NHS Foundation Trust, Lancaster, UK

2 Lotus Eye Hospital and Institute, Avinashi Road, Coimbatore, TN, India

3 University Vita-Salute, Scientific Institute San Raffaele, Milano, Italy

4 Gavin Herbert Eye Institute, University of California, Irvine, CA, USA

5 Division of Ophthalmology, Tel Aviv Sourasky Medical Center and Sackler Faculty of Medicine, Tel Aviv University, Tel Aviv, Israel

\section{Opportunities of the S-ROP imaging}

The greatest boon of the S-ROP screening has been its cost effectiveness. Policy makers would love the option of SROP screening given its low-cost, one-time investment, nil maintenance and repairs. With financial constraints in the lower and middle-income economies, any investment in ROP screening and imaging is considered an additional and un-called burden that could only serve a small population. In total, 3.5 million babies are born premature in India alone and the scale of the problem is much higher in the SubSaharan Africa [9]. Given the magnitude of ROP-related blindness in the developing world, any investment towards ROP screening should be considered imperative and of vital health-care importance. It is of general belief amongst the hospital management that the limited resources instead could be more efficiently utilised in other areas of ophthalmic practice that could subserve a larger population such as management of cataract blindness or due to DR and Glaucoma. S-ROP is the only feasible solution in terms of health economics at the moment for this problem. S-ROP screening also facilitates tele-ophthalmology with real-time transfer of images to a treating referral centre, thanks to the various apps that support these functions. Hence, S-ROP could be easily carried out in the peripheral units in remote and rural settings with a team of non-ophthalmic personnel such as technicians or nurses trained in image acquisition. There are no logistical barriers of moving a heavy, large piece of equipment by the screening team. The procedure is also considered to be less distressing for the baby as it is a non-contact procedure. This also minimises any risk of infection, as it does not involve any imaging probes. We have published a similar pilot model in the recent past [10]. Furthermore, it also allows for the imaging to be carried out in lower light exposure when compared with an indirect ophthalmoscope [11].

The opportunities for future developments lie in the fact that S-ROP acquired images could easily be integrated with 
any $\mathrm{AI}$ algorithms and train the system to recognise and triage according to the stage of ROP with focussed machine-learning $[5,6]$. This could potentially reduce the clinician's involvement at every stage of ROP management, making way for more efficient use of the trained paediatric ophthalmologists only when their expertise is inevitably needed.

The 3D printing technology has opened up options to develop custom-made retinal imaging adaptors for any smartphone or digital camera [7, 8]. Once developed, these adaptors last long and could be an indispensable resource in a non-institutional or solo practice, where financial strain could be a persistent limiting factor.

\section{Challenges with the S-ROP imaging}

A low-cost, low-investment tool comes with its own limitations. The foremost challenge with S-ROP screening has been in obtaining high-quality images. The difficulty, although not directly related to the device, has largely been due to lack of experience and expertise in technical aspects of the image acquisition. Adequate, well-supervised training modules to improve the operator confidence in obtaining consistently good images in a short time frame could overcome this. The optimal placement of the condensing lens system, co-axial with the camera's field of view is a skill that needs mastering to obtain and reproduce excellent images.

Another issue that needs addressing is related to the width of imaging. The field of view with smartphone photography such as MII Ret Cam has been around $53^{\circ}$ using a $28 \mathrm{D}$ lens, this however, could be pushed up to $90^{\circ}$ with the use of a $40 \mathrm{D}$ lens [12]. ROP screening ideally requires imaging zones 2 and 3, both for diagnosis and monitoring the response to treatment. However, this cannot be a restraining factor per se, as the plus disease that determines the treatment could be ascertained with posterior pole visualisation and imaging which any of the S-ROP screening devices would facilitate [13]. Although the SROP screening models including Smart ROP initiative have managed to obtain satisfactory imaging, there is still a need to develop a better system to capture wide-field imaging (WFI). The width of imaging offered by the Retcam 2 is up to $130^{\circ}$ and the Optos WFI, up to $200^{\circ}[14,15]$.

Smartphone retinal photography is in its infancy but with advances in the field of imaging devices and smart phones, it is a tool that is here to stay. The opportunity that it could offer for lower- and middle-income countries is enormous despite its obvious limitations. The challenges it poses could in itself be an impetus for further developments and innovations.

\section{Compliance with ethical standards}

Conflict of interest AS: Consultant: for Novartis, Allergan, Bayer and Intas. Proprietary interest in MII Ret Cam. FB: Consultant: Allergan, Bayer, Boehringer-Ingelheim, Fidia Sooft, Hofmann La Roche, Novartis, NTC Pharma, Sifi, Thrombogenics, Zeiss. BDK: Clinical research: Alcon, Alimera, Allegro, Allergan, Apellis, Clearside, Genentech, GSK, Ionis, jCyte, Novartis, Regeneron, ThromboGenics; Consultant: Alimera, Allegro, Allergan, Cell Care, Dose, Eyedaptic, Galimedix, Genentech, Glaukos, Interface Biologics, jCyte, Novartis, Ophthotech, Regeneron, Revana, Theravance Biopharma. AL reports other from Allergan, other from Novartis, other from Roche, other from Notal Vision, other from Forsight labs, other from Beyeonics, other from Bayer Health Care. The remaining authors declare that they have no conflict of interest.

Publisher's note Springer Nature remains neutral with regard to jurisdictional claims in published maps and institutional affiliations.

\section{References}

1. Lekha T, Ramesh S, Sharma AAG. MII RetCam assisted smartphone-based fundus imaging for retinopathy of prematurity. Indian J Ophthalmol. 2019;67:834-9.

2. Sharma A, Subramaniam SD, Ramachandran KI, Lakshmikanthan C, Krishna S, Sundaramoorthy SK. Smartphone-based fundus camera device (MII Ret Cam) and technique with ability to image peripheral retina. Eur J Ophthalmol. 2016;26:142-4.

3. Bilong Y, Domngang CN, Nwanlih Gimma G, Katte J-C, Afetane ET, Kagmeni G, Mbanya JC, Kumar N, Sharma A, Sobngwi E. Smartphone-assisted glaucoma screening in patients with type 2 diabetes: a pilot study. Med Hypothesis Discov Innov Ophthalmol. 2020;9:61-65.

4. Sharma A, Bilong Y, Patil J, Katte C, et al. MIIRetCam (Make In India Retina Camera) assisted retinal imaging in paediatric patients: useful, artefacts, learning curve. J Fr Ophtalmol. 2020;43:e35-8.

5. Ataer-Cansizoglu E, Bolon-Canedo V, Campbell JP, Bozkurt A, Erdogmus D, Kalpathy-Cramer J, et al. i-ROP Research Consortium Computer-based image analysis for plus disease diagnosis in retinopathy of prematurity performance of the "i-ROP" system and image features associated with expert diagnosis. Transl Vis Sci Technol. 2015;4:5.

6. Bolón-Canedo V, Ataer-Cansizoglu E, Erdogmus D, KalpathyCramer J, Fontenla-Romero O, Alonso-Betanzos A, et al. Dealing with inter-expert variability in retinopathy of prematurity: a machine learning approach. Comput Methods Prog Biomed. 2015;122:1-15.

7. Kopparapu K, Kopparapu N, Zhang J. Inexpensive mobile diagnosis of diabetic retinopathy using deep learning. Society For Science \& The Public. 2017. https://abstracts.societyforscience. org/Home/FullAbstract?ProjectId=8946.

8. Hong SC. 3D printable retinal imaging adapter for smartphones could go global. Graefes Arch Clin Exp Ophthalmol. 2015;253:1831-3.

9. Project operational guidelines. Prevention of blindness from retinopathy of prematurity in neonatal care units. https://phfi.org/wpcontent/uploads/2019/05/2018-ROP-operationalguidelines.pdf. Accessed 27 Mar 2020.

10. Sharma A, Goyal A, Bilong Y, Shah P, Banker A, Kumar N Comparison of a Smartphone-based photography method with indirect ophthalmoscopic assessment in referable retinopathy of 
prematurity: a smart retinopathy of prematurity model pilot study. Ophthalmol Retina 2019;3:911-2.

11. Kim DY, Delori F, Mukai S. Smartphone photography safety. Ophthalmology. 2012;119:2200-1.

12. Goyal A, Gopalakrishnan M, Anantharaman G, Chandrashekharan D, Thachil T, Sharma A. Smartphone guided wide-field imaging for retinopathy of prematurity in neonatal intensive care unit-a Smart ROP (SROP) initiative. Indian J Ophthalmol. 2019;67:840-5.
13. Adams GGW. ROP in Asia. Eye 2020;34:607-8.

14. Richter GM, Williams SL, Starren J, Flynn JT, Chiang MF. Telemedicine for retinopathy of prematurity diagnosis: evaluation and challenges. Surv Ophthalmol. 2009;54:671-85.

15. Patel CK, Fung THM, Muqit MMK, Mordant DJ, Brett J, Smith L, et al. Non-contact ultra-widefield imaging of retinopathy of prematurity using the Optos dual wavelength scanning laser ophthalmoscope. Eye. 2013;27:589-96. 